\title{
Article
}

\section{A differential inequality and meromorphic starlike and convex functions}

\section{Kuldeep Kaur Shergill ${ }^{1, *}$ and Sukhwinder Singh Billing ${ }^{2}$}

1 Department of Mathematics, Sri Guru Granth Sahib World University, Fatehgarh Sahib-140407(Punjab), India.

* Correspondence: kkshergill16@gmail.com

Received: 22 July 2020; Accepted: 25 August 2020; Published: 8 October 2020.

Abstract: In the present paper, we study a differential inequality involving certain differential operator. As a special case of our main result, we obtained certain differential inequalities implying sufficient conditions for meromorphic starlike and meromorphic convex functions of certain order.

Keywords: Meromorphic function, meromorphic starlike function, meromorphic convex function, multivalent function, differential operator.

MSC: 30C45, 30C80.

\section{Introduction}

$\mathbf{L}$

et $\Sigma_{p, n}$ denote the class of functions of the form

$$
f(z)=\frac{a_{-1}}{z^{p}}+\sum_{k=n}^{\infty} a_{k-p} z^{k-p}(p, n \in \mathbb{N}=\{1,2,3, \ldots\}),
$$

which are analytic and $p$-valent in the punctured unit disc $\mathbb{E}_{0}=\mathbb{E} \backslash\{0\}$, where $\mathbb{E}=\{z \in \mathbb{C}:|z|<1\}$. Define

$$
\begin{aligned}
& D^{0} f(z)=f(z) \\
& D^{1} f(z)=\frac{a_{-1}}{z^{p}}+2 a_{0}+3 a_{1} z+4 a_{2} z^{2}+\ldots=\frac{\left(z^{2} f(z)\right)^{\prime}}{z}, \\
& D^{2} f(z)=D^{1}\left(D^{1} f(z)\right),
\end{aligned}
$$

and for $n=1,2,3, \ldots$

$$
D^{n} f(z)=D^{1}\left(D^{n-1} f(z)\right)=\frac{\left(z^{2} D^{n-1} f(z)\right)^{\prime}}{z} .
$$

Let $\mathcal{M S}_{n}^{*}(p, \alpha)$ denote the class of functions $f \in \Sigma_{p, n}$ if

$$
-\Re \frac{1}{p}\left(\frac{D^{n+1} f(z)}{D^{n} f(z)}-2\right)>\alpha,(\alpha<1 ; z \in \mathbb{E}) .
$$

and let $\mathcal{M K}_{n}(p, \alpha)$ denote the class of functions $f \in \Sigma_{p, n}$ if

$$
-\Re \frac{1}{p}\left(\frac{\left(D^{n+1} f(z)\right)^{\prime}}{\left(D^{n} f(z)\right)^{\prime}}-2\right)>\alpha,(\alpha<1 ; z \in \mathbb{E}) .
$$

The classes of meromorphic starlike functions of order $\alpha$ and meromorphic convex functions of order $\alpha$ are denoted by $\mathcal{M S}^{*}(\alpha)$ and $\mathcal{M K}(\alpha)$, respectively and are defined as:

$$
\mathcal{M S}^{*}(\alpha)=\left\{f \in \Sigma:-\Re\left(\frac{z f^{\prime}(z)}{f(z)}\right)>\alpha,(0 \leq \alpha<1 ; z \in \mathbb{E})\right\},
$$


and

$$
\mathcal{M K}(\alpha)=\left\{f \in \Sigma:-\Re\left(1+\frac{z f^{\prime \prime}(z)}{f^{\prime}(z)}\right)>\alpha,(0 \leq \alpha<1 ; z \in \mathbb{E})\right\} .
$$

Note that $\mathcal{M S}^{*}(\alpha)=\mathcal{M S}_{0}^{*}(1, \alpha)$ and $\mathcal{M K}(\alpha)=\mathcal{M K}_{0}(1, \alpha)$. In the theory of meromorphic functions, there exists a variety of results for starlikeness and convexity of meromorphic functions, we state some of them below. Wang et al. [1] proved the following results;

Theorem 1. If $f(z) \in \Sigma_{p}$ satisfies the following inequality

$$
\left|\frac{f(z)}{z f^{\prime}(z)}\left(1+\frac{z f^{\prime \prime}(z)}{f^{\prime}(z)}-\frac{z f^{\prime}(z)}{f(z)}\right)\right|<\mu\left(0<\mu<\frac{1}{p}\right)
$$

then $f \in \mathcal{M S}_{p}^{*}\left(\frac{p}{1+p \mu}\right)$.

Theorem 2. If $f(z) \in \Sigma_{p}$ satisfies the inequality

$$
\left|\frac{z f^{\prime}(z)}{f(z)}-\frac{z f^{\prime \prime}(z)}{f^{\prime}(z)}-1\right|<\delta(0<\delta<1)
$$

then $f \in \mathcal{M S}_{p}^{*}(p(1-\delta))$.

Theorem 3. If $f(z) \in \Sigma_{p}$ satisfies the following inequality

$$
\Re\left(\frac{z f^{\prime}(z)}{f(z)}+\beta \frac{z^{2} f^{\prime \prime}(z)}{f(z)}\right)<\beta \lambda\left(\lambda+\frac{1}{2}\right)+\frac{1}{2} p \beta-\lambda \quad\left(\beta \geq 0, p-\frac{1}{2} \leq \lambda \leq p\right),
$$

then $f \in \mathcal{M S}_{p}^{*}(\lambda)$.

Goswami et al. [2] proved the following results;

Theorem 4. If $f(z) \in \Sigma_{p}, n$ with $f(z) \neq 0$ for all $z \in \mathbb{E}_{0}$, satisfies the following inequality

$$
\left|\left[z^{p} f(z)\right]^{\frac{1}{\alpha-p}}\left(\frac{z f^{\prime}(z)}{f(z)}+\alpha\right)+p-\alpha\right|<\frac{(n+1)(p-\alpha)}{\sqrt{(n+1)^{2}+1}}, z \in \mathbb{E},
$$

for some real values of $\alpha(0 \leq \alpha<p)$, then $f \in \mathcal{M S}_{p, n}^{*}(\alpha)$.

Theorem 5. If $f(z) \in \Sigma_{p}, n$ with $f(z) \neq 0$ for all $z \in \mathbb{E}_{0}$ satisfies the following inequality

$$
\left|\frac{\gamma\left[z^{p} f(z)\right]^{\gamma}}{z}\left(\frac{z f^{\prime}(z)}{f(z)}+p\right)\right| \leq \frac{(n+1)}{2 \sqrt{(n+1)^{2}+1}}, z \in \mathbb{E},
$$

for $\gamma \leq-\frac{1}{p}$, then $f \in \mathcal{M S}_{p, n}^{*}\left(p+\frac{1}{\gamma}\right)$.

Theorem 6. If $f(z) \in \Sigma_{p}, n$ with $f(z) \neq 0$ for all $z \in \mathbb{E}_{0}$, satisfies the inequality

$$
\left|\left(\frac{z^{p+1} f^{\prime}(z)}{-p}\right)^{\frac{1}{\alpha-p}}\left(1+\frac{z f^{\prime \prime}(z)}{f^{\prime}(z)}+\alpha\right)+p-\alpha\right|<\frac{(n+1)(p-\alpha)}{\sqrt{(n+1)^{2}+1}}, z \in \mathbb{E},
$$

for some real values of $\alpha(0 \leq \alpha<p)$, then $f \in \mathcal{M} \mathcal{K}_{p, n}(\alpha)$. 
Theorem 7. If $f(z) \in \Sigma_{p}$, nwith $f(z) \neq 0$ for all $z \in \mathbb{E}_{0}$, satisfies the inequality

$$
\left|\frac{1}{z}\left(\frac{z^{p+1} f^{\prime}(z)}{-p}\right)^{\frac{1}{\alpha-p}}\left(1+\frac{z f^{\prime \prime}(z)}{f^{\prime}(z)}+p\right)\right| \leq \frac{(n+1)(p-\alpha)}{2 \sqrt{(n+1)^{2}+1}}, z \in \mathbb{E},
$$

for some real values of $\alpha(0 \leq \alpha<p)$, then $f \in \mathcal{M K}_{p, n}(\alpha)$.

From above stated results, we notice that a number of sufficient conditions for meromorphic starlike and meromorphic convex functions have been obtained in terms of differential inequalities in the literature of meromorphic functions. The study of such results is a source of motivation for us to produce the present paper. In the present paper, we study differential inequalities involving a differential operator. As particular cases of our main results, we derive certain sufficient conditions for meromorphic starlike and meromorphic convex functions.

\section{Preliminaries}

We shall use the following lemma of [3] to prove our result.

Lemma 1. Suppose $w$ is a nonconstant analytic function in $\mathbb{E}$ with $w(0)=0$. If $|w(z)|$ attains its maximum value at a point $z_{0} \in \mathbb{E}$ on the circle $|z|=r<1$, then $z_{0} w^{\prime}\left(z_{0}\right)=m w\left(z_{0}\right)$, where $m \geq 1$, is some real number.

\section{Main Results}

Theorem 8. Let $f(z) \in \Sigma_{p}$ satisfy

$$
\left|\frac{D^{n+1}[f](z)}{D^{n}[f](z)}-1\right|^{\gamma}\left|\frac{D^{n+2}[f](z)}{D^{n+1}[f](z)}-1\right|^{\beta}<M(p, \alpha, \beta, \gamma), z \in \mathbb{E},
$$

for some real numbers $\alpha, \beta$ and $\gamma$ such that $0 \leq \alpha<p, \beta \geq 0, \gamma \geq 0, \beta+\gamma>0$, then $f(z) \in \mathcal{M S}_{n}^{*}(p, \alpha)$, where $n \in \mathbb{N}_{0}=\mathbb{N} \cup\{0\}$ and

$$
M(p, \alpha, \beta, \gamma)=\left\{\begin{array}{l}
\left(1-\frac{\alpha}{p}\right)^{\gamma}\left(\frac{1}{2}-\frac{\alpha}{p}\right)^{\beta}, 0 \leq \alpha<\frac{p}{2} \\
\left(1-\frac{\alpha}{p}\right)^{\gamma+\beta}\left(\frac{2}{2-\frac{\alpha}{p}}\right)^{\beta}, \frac{p}{2} \leq \alpha<p
\end{array}\right.
$$

Proof. We consider the following two cases separately.

Case (i). When $0 \leq \alpha<\frac{p}{2}$. Writing $\frac{\alpha}{p}=\mu$, we see that $0 \leq \mu<\frac{1}{2}$. Define a function $w$ as

$$
2-\frac{D^{n+1}[f](z)}{D^{n}[f](z)}=\frac{1+(1-2 \mu) w(z)}{1-w(z)}
$$

where $w$ is an analytic function in $\mathbb{E}, w(0)=0$ and $w(z) \neq 1$ in $\mathbb{E}$. Differentiating (2) logarithmatically, we get

$$
\frac{z\left(D^{n+1}[f](z)\right)^{\prime}}{D^{n+1}[f](z)}-\frac{z\left(D^{n}[f](z)\right)^{\prime}}{D^{n}[f](z)}=\frac{2(\mu-1) z w^{\prime}(z)}{(1-w(z))(1+(2 \mu-3) w(z))} .
$$

From the definition, we have the identity

$$
z\left(D^{n}[f](z)\right)^{\prime}=D^{n+1}[f](z)-2 D^{n}[f](z)
$$


Using (4) in (3), we get $\frac{D^{n+2}[f](z)}{D^{n+1}[f](z)}=\frac{1+(2 \mu-3) w(z)}{1-w(z)}+\frac{2(\mu-1) z w^{\prime}(z)}{(1-w(z))(1+(2 \mu-3) w(z))}$. So, we have

$$
\begin{aligned}
\left|\frac{D^{n+1}[f](z)}{D^{n}[f](z)}-1\right|^{\gamma}\left|\frac{D^{n+2}[f](z)}{D^{n+1}[f](z)}-1\right|^{\beta} & =\left|\frac{2(1-\mu) w(z)}{1-w(z)}\right|^{\gamma}\left|\frac{2(1-\mu) w(z)}{1-w(z)}+\frac{2(1-\mu) z w^{\prime}(z)}{(1-w(z))(1+(2 \mu-3) w(z))}\right|^{\beta} \\
& =\left|\frac{2(1-\mu) w(z)}{1-w(z)}\right|^{\gamma+\beta}\left|1+\frac{z w^{\prime}(z)}{w(z)(1+(2 \mu-3) w(z))}\right|^{\beta}
\end{aligned}
$$

We claim that $|w(z)|<1, z \in \mathbb{E}$. Suppose, to the contrary, that there exists a point $z_{0} \in \mathbb{E}$ such that $\max _{|z| \leq\left|z_{0}\right|}|w(z)|=\left|w\left(z_{0}\right)\right|=1$. Then by Lemma 1, we have $w\left(z_{0}\right)=e^{i \theta}, 0 \leq \theta<2 \pi$ and $z_{0} w^{\prime}\left(z_{0}\right)=$ $m w\left(z_{0}\right), m \geq 1$. Therefore

$$
\begin{aligned}
\left|\frac{D^{n+1}[f](z)}{D^{n}[f](z)}-1\right|^{\gamma}\left|\frac{D^{n+2}[f](z)}{D^{n+1}[f](z)}-1\right|^{\beta} & =\left|\frac{2(1-\mu) w\left(z_{0}\right)}{1-w\left(z_{0}\right)}\right|^{\gamma+\beta}\left|1+\frac{m}{1+(2 \mu-3) w\left(z_{0}\right)}\right|^{\beta} \\
& =\frac{2^{\gamma+\beta}(1-\mu)^{\gamma+\beta}}{\left|1-e^{i \theta}\right| \gamma+\beta}\left|1+\frac{m}{1+(2 \mu-3) e^{i \theta}}\right|^{\beta} \\
& \geq(1-\mu)^{\gamma+\beta}\left(1-\frac{m}{2(1-\mu)}\right)^{\beta} \\
& \geq(1-\mu)^{\gamma+\beta}\left(1-\frac{1}{2(1-\mu)}\right)^{\beta} \\
& =(1-\mu)^{\gamma}\left(\frac{1}{2}-\mu\right)^{\beta},
\end{aligned}
$$

which contradicts (1) for $0 \leq \alpha<\frac{p}{2}$. Therefore we must have $|w(z)|<1$ for all $z \in \mathbb{E}$, and hence from (2), we conclude that $f \in \mathcal{M S}_{n}^{*}(p, \alpha)$.

Case (ii). When $\frac{p}{2} \leq \alpha<p$, therefore we must have $\frac{1}{2} \leq \mu<1$, where $\mu=\frac{\alpha}{p}$. Let $w$ be defined by

$$
2-\frac{D^{n+1}[f](z)}{D^{n}[f](z)}=\frac{\mu}{\mu-(1-\mu) w(z)},
$$

where $w(z) \neq \frac{\mu}{1-\mu}$ in $\mathbb{E}$. Then $\mathrm{w}$ is analytic in $\mathbb{E}$ with $w(0)=0$. Proceeding as in Case (i) above, we obtain

$$
\begin{aligned}
& \left|\frac{D^{n+1}[f](z)}{D^{n}[f](z)}-1\right|^{\gamma}\left|\frac{D^{n+2}[f](z)}{D^{n+1}[f](z)}-1\right|^{\beta} \\
= & \left|\frac{(1-\mu) w(z)}{\mu-(1-\mu) w(z)}\right|^{\gamma}\left|\frac{(1-\mu) w(z)}{\mu-(1-\mu) w(z)}+\frac{\mu(1-\mu) z w^{\prime}(z)}{(\mu-(1-\mu) w(z))(\mu-2(1-\mu) w(z))}\right|^{\beta} \\
= & \left|\frac{1-\mu}{\mu-(1-\mu) w(z)}\right|^{\gamma+\beta}|w(z)|^{\gamma}\left|w(z)+\frac{\mu z w^{\prime}(z)}{\mu-2(1-\mu) w(z)}\right|^{\beta} .
\end{aligned}
$$

We shall prove that $|w(z)|<1, z \in \mathbb{E}$. If not, suppose there exists a point $z_{0} \in \mathbb{E}$ such that there exists a point $z_{0} \in \mathbb{E}$ such that $\max _{|z| \leq\left|z_{0}\right|}|w(z)|=\left|w\left(z_{0}\right)\right|=1$. Then by Lemma 1 we have $w\left(z_{0}\right)=e^{i \theta}, 0 \leq \theta<2 \pi$ and $z_{0} w^{\prime}\left(z_{0}\right)=m w\left(z_{0}\right), m \geq 1$. Therefore

$$
\begin{aligned}
\left|\frac{D^{n+1}[f](z)}{D^{n}[f](z)}-1\right|^{\gamma}\left|\frac{D^{n+2}[f](z)}{D^{n+1}[f](z)}-1\right|^{\beta} & =\frac{(1-\mu)^{\gamma+\beta}\left(1+\frac{m \mu}{\mu+2-2 \mu}\right)^{\beta}}{\left|\mu-(1-\mu) w\left(z_{0}\right)\right|^{\gamma+\beta}} \\
& \geq(1-\mu)^{\gamma+\beta}\left(\frac{2}{2-\mu}\right)^{\beta}
\end{aligned}
$$

which contradicts (1) for $\frac{p}{2} \leq \alpha<p$. Therefore, we must have $|w(z)|<1$ for all $z \in \mathbb{E}$, and hence in view of (6), we conclude that $f \in \mathcal{M S}_{n}^{*}(p, \alpha)$. This completes the proof of the theorem. 
Theorem 9. Let $f(z) \in \Sigma_{p}$ satisfy

$$
\left|\frac{\left(D^{n+1}[f](z)\right)^{\prime}}{\left(D^{n}[f](z)\right)^{\prime}}-1\right|^{\gamma}\left|\frac{\left(D^{n+2}[f](z)\right)^{\prime}}{\left(D^{n+1}[f](z)\right)^{\prime}}-1\right|^{\beta}<M(p, \alpha, \beta, \gamma), z \in \mathbb{E},
$$

for some real numbers $\alpha, \beta$ and $\gamma$ such that $0 \leq \alpha<p, \beta \geq 0, \gamma \geq 0, \beta+\gamma>0$, then $f(z) \in \mathcal{M} \mathcal{K}_{n}(p, \alpha)$, where $n \in \mathbb{N}_{0}=\mathbb{N} \cup\{0\}$ and

$$
M(p, \alpha, \beta, \gamma)=\left\{\begin{array}{l}
\left(1-\frac{\alpha}{p}\right)^{\gamma}\left(\frac{1}{2}-\frac{\alpha}{p}\right)^{\beta}, 0 \leq \alpha<\frac{p}{2} \\
\left(1-\frac{\alpha}{p}\right)^{\gamma+\beta}\left(\frac{2}{2-\frac{\alpha}{p}}\right)^{\beta}, \frac{p}{2} \leq \alpha<p
\end{array}\right.
$$

Proof. Again,we consider the following two cases separately.

Case (i). When $0 \leq \alpha<\frac{p}{2}$. Writing $\frac{\alpha}{p}=\mu$, we see that $0 \leq \mu<\frac{1}{2}$. Define a function $w$ as

$$
2-\frac{\left(D^{n+1}[f](z)\right)^{\prime}}{\left(D^{n}[f](z)\right)^{\prime}}=\frac{1+(1-2 \mu) w(z)}{1-w(z)}
$$

where $w$ is an analytic function in $\mathbb{E}, w(0)=0$ and $w(z) \neq 1$ in $\mathbb{E}$. Differentiating (9) logarithmatically, we get

$$
\frac{z\left(D^{n+1}[f](z)\right)^{\prime \prime}}{\left(D^{n+1}[f](z)\right)^{\prime}}-\frac{z\left(D^{n}[f](z)\right)^{\prime \prime}}{\left(D^{n}[f](z)\right)^{\prime}}=\frac{2(\mu-1) z w^{\prime}(z)}{(1-w(z))(1+(2 \mu-3) w(z))} .
$$

From the following identity

$$
z\left(D^{n}[f](z)\right)^{\prime}=D^{n+1}[f](z)-2 D^{n}[f](z)
$$

we have

$$
z\left(D^{n}[f](z)\right)^{\prime \prime}=\left(D^{n+1}[f](z)\right)^{\prime}-3\left(D^{n}[f](z)\right)^{\prime} .
$$

Using the identity (12), Equation (10) may be written as

$$
\frac{\left(D^{n+2}[f](z)\right)^{\prime}}{\left(D^{n+1}[f](z)\right)^{\prime}}=\frac{1+(2 \mu-3) w(z)}{1-w(z)}+\frac{2(\mu-1) z w^{\prime}(z)}{(1-w(z))(1+(2 \mu-3) w(z))} .
$$

So, we have

$$
\begin{aligned}
& \left|\frac{\left(D^{n+1}[f](z)\right)^{\prime}}{\left(D^{n}[f](z)\right)^{\prime}}-1\right|^{\gamma}\left|\frac{\left(D^{n+2}[f](z)\right)^{\prime}}{\left(D^{n+1}[f](z)\right)^{\prime}}-1\right|^{\beta} \\
= & \left|\frac{2(1-\mu) w(z)}{1-w(z)}\right|^{\gamma}\left|\frac{2(1-\mu) w(z)}{1-w(z)}+\frac{2(1-\mu) z w^{\prime}(z)}{(1-w(z))(1+(2 \mu-3) w(z))}\right|^{\beta} \\
= & \left|\frac{2(1-\mu) w(z)}{1-w(z)}\right|^{\gamma+\beta}\left|1+\frac{z w^{\prime}(z)}{w(z)(1+(2 \mu-3) w(z))}\right|^{\beta} .
\end{aligned}
$$

The remaining part of the proof is similar to that of Theorem 8.

\section{Criteria for Starlikeness and Convexity}

When we assign particular values to various parameters involved in Theorem 8 and Theorem 9, we obtain following special cases. Setting $n=0$ in Theorem 8 , we obtain the following result. 
Corollary 1. Let $f \in \Sigma_{p}$ satisfy the condition

$$
\left|\frac{1}{p}\left(\frac{z f^{\prime}(z)}{f(z)}\right)-1\right|^{\gamma}\left|\frac{1}{p}\left(\frac{z f^{\prime \prime}(z)+3 f^{\prime}(z)}{z f^{\prime}(z)+2 f(z)}\right)-1\right|^{\beta}<\left\{\begin{array}{l}
\left(1-\frac{\alpha}{p}\right)^{\gamma}\left(\frac{1}{2}-\frac{\alpha}{p}\right)^{\beta}, 0 \leq \alpha<\frac{p}{2} \\
\left(1-\frac{\alpha}{p}\right)^{\gamma+\beta}\left(\frac{2}{2-\frac{\alpha}{p}}\right)^{\beta}, \frac{p}{2} \leq \alpha<p,
\end{array}\right.
$$

for all $z \in \mathbb{E}$ and for some real numbers $\alpha(0 \leq \alpha<1), \beta \geq 0$ and $\gamma \geq 0$ with $\beta+\gamma>0$, then $f \in \mathcal{M S}^{*}(p, \alpha)$.

For $p=1$, Theorem 8 reduces to the following;

Corollary 2. For some real numbers $\alpha(0 \leq \alpha<1), \beta \geq 0$ and $\gamma \geq 0$ with $\beta+\gamma>0$, if $f \in \Sigma$ satisfies

$$
\left|\frac{D^{n+1}[f](z)}{D^{n}[f](z)}-1\right|^{\gamma}\left|\frac{D^{n+2}[f](z)}{D^{n+1}[f](z)}-1\right|^{\beta}<\left\{\begin{array}{l}
(1-\alpha)^{\gamma}\left(\frac{1}{2}-\alpha\right)^{\beta}, 0 \leq \alpha<\frac{1}{2}, \\
(1-\alpha)^{\gamma+\beta}\left(\frac{2}{2-\alpha}\right)^{\beta}, \frac{1}{2} \leq \alpha<1,
\end{array}\right.
$$

in $\mathbb{E}$, then $\mathcal{M S}_{n}^{*}(1, \alpha)$, where $n \in \mathbb{N}_{0}$.

Setting $n=0$ in above corollary, yields the following result.

Corollary 3. Let $f(z) \in \Sigma$ satisfy the condition

$$
\left|\frac{z f^{\prime}(z)}{f(z)}+1\right|^{\gamma}\left|\frac{z f^{\prime \prime}(z)+3 f^{\prime}(z)}{z f^{\prime}(z)+2 f(z)}-1\right|^{\beta}<\left\{\begin{array}{l}
(1-\alpha)^{\gamma}\left(\frac{1}{2}-\alpha\right)^{\beta}, 0 \leq \alpha<\frac{1}{2}, \\
(1-\alpha)^{\gamma+\beta}\left(\frac{2}{2-\alpha}\right)^{\beta}, \frac{1}{2} \leq \alpha<1,
\end{array}\right.
$$

where $z \in \mathbb{E}, \alpha(0 \leq \alpha<1), \beta \geq 0$ and $\gamma \geq 0$ with $\beta+\gamma>0$, then $f \in \mathcal{M S}^{*}(\alpha)$.

Setting $\beta=\gamma=1$ and $\alpha=0$ in above corollary, we obtain the following result.

Remark 1. If $f(z) \in \Sigma$ satisfies

$$
\left|\frac{z f^{\prime}(z)}{f(z)}+1\right|\left|\frac{z f^{\prime \prime}(z)+3 f^{\prime}(z)}{z f^{\prime}(z)+2 f(z)}-1\right|<\frac{1}{2}, z \in \mathbb{E},
$$

then $f \in \mathcal{M S}^{*}$.

By writing $\beta=1$ and $\gamma=0$, Theorem 8 , we get

Corollary 4. If for all $z \in \mathbb{E}$, a function $f \in \Sigma_{p}$ satisfies the condition

$$
\frac{D^{n+2}[f](z)}{D^{n+1}[f](z)} \prec\left\{\begin{array}{l}
1+\left(\frac{1}{2}-\frac{\alpha}{p}\right) z, 0 \leq \alpha<\frac{p}{2}, \\
1+\left[\frac{2\left(1-\frac{\alpha}{p}\right)}{2-\frac{\alpha}{p}}\right] z, \frac{p}{2} \leq \alpha<p,
\end{array}\right.
$$

then

$$
2-\frac{D^{n+1}[f](z)}{D^{n}[f](z)} \prec \frac{1+\left(1-\frac{2 \alpha}{p}\right) z}{1-z}, z \in \mathbb{E},
$$

i.e.

$$
\Re\left(2-\frac{D^{n+1}[f](z)}{D^{n}[f](z)}\right)>\frac{\alpha}{p} .
$$

Setting $n=0$ in Theorem 9, we obtain the following result.

Corollary 5. Let $f \in \Sigma_{p}$ satisfy the condition 


$$
\left|\frac{1}{p}\left(\frac{z f^{\prime \prime}(z)+3 f^{\prime}(z)}{f^{\prime}(z)}\right)-1\right|^{\gamma}\left|\frac{1}{p}\left(\frac{z^{2} f^{\prime \prime \prime}(z)+7 z f^{\prime}(z)+9 f^{\prime}(z)}{z f^{\prime \prime}(z)+3 f(z)}\right)-1\right|^{\beta}<\left\{\begin{array}{l}
\left(1-\frac{\alpha}{p}\right)^{\gamma}\left(\frac{1}{2}-\frac{\alpha}{p}\right)^{\beta}, 0 \leq \alpha<\frac{p}{2}, \\
\left(1-\frac{\alpha}{p}\right)^{\gamma+\beta}\left(\frac{2}{2-\frac{\alpha}{p}}\right)^{\beta}, \frac{p}{2} \leq \alpha<p,
\end{array}\right.
$$

for all $z \in \mathbb{E}$ and for some real numbers $\alpha(0 \leq \alpha<1), \beta \geq 0$ and $\gamma \geq 0$ with $\beta+\gamma>0$, then $f \in \mathcal{M K}(p, \alpha)$.

For $p=1$, Theorem 9 reduces to the following;

Corollary 6. For some real numbers $\alpha(0 \leq \alpha<1), \beta \geq 0$ and $\gamma \geq 0$ with $\beta+\gamma>0$, if $f \in \Sigma$ satisfies

$$
\left|\frac{\left(D^{n+1}[f](z)\right)^{\prime}}{\left(D^{n}[f](z)\right)^{\prime}}-1\right|^{\gamma}\left|\frac{\left(D^{n+2}[f](z)\right)^{\prime}}{\left(D^{n+1}[f](z)\right)^{\prime}}-1\right|^{\beta}<\left\{\begin{array}{l}
(1-\alpha)^{\gamma}\left(\frac{1}{2}-\alpha\right)^{\beta}, 0 \leq \alpha<\frac{1}{2}, \\
(1-\alpha)^{\gamma+\beta}\left(\frac{2}{2-\alpha}\right)^{\beta}, \frac{1}{2} \leq \alpha<1,
\end{array}\right.
$$

in $\mathbb{E}$, then $\mathcal{M K}_{n}(1, \alpha)$, where $n \in \mathbb{N}_{0}$.

Setting $n=0$ in above corollary, yields the following result;

Corollary 7. Let $f(z) \in \Sigma$ satisfy the condition

$$
\left|\frac{z f^{\prime \prime}(z)}{f^{\prime}(z)}+2\right|^{\gamma}\left|\frac{z^{2} f^{\prime \prime \prime}(z)+7 z f^{\prime \prime}(z)+9 f^{\prime}(z)}{z f^{\prime \prime}(z)+3 f(z)}-1\right|^{\beta}<\left\{\begin{array}{l}
(1-\alpha)^{\gamma}\left(\frac{1}{2}-\alpha\right)^{\beta}, 0 \leq \alpha<\frac{1}{2}, \\
(1-\alpha)^{\gamma+\beta}\left(\frac{2}{2-\alpha}\right)^{\beta}, \frac{1}{2} \leq \alpha<1,
\end{array}\right.
$$

where $z \in \mathbb{E}, \alpha(0 \leq \alpha<1), \beta \geq 0$ and $\gamma \geq 0$ with $\beta+\gamma>0$, then $f \in \mathcal{M K}(\alpha)$.

Setting $\beta=\gamma=1$ and $\alpha=0$ in above corollary, we obtain the following result;

Remark 2. If $f(z) \in \Sigma$ satisfies

$$
\left|\frac{z f^{\prime \prime}(z)}{f^{\prime}(z)}+2\right|\left|\frac{z^{2} f^{\prime \prime \prime}(z)+7 z f^{\prime \prime}(z)+9 f^{\prime}(z)}{z f^{\prime \prime}(z)+3 f(z)}-1\right|<\frac{1}{2}, z \in \mathbb{E},
$$

then $f \in \mathcal{M K}$.

Acknowledgments: The authors are grateful to the referee for careful reading of the paper and valuable suggestions and comments

Author Contributions: All authors contributed equally to the writing of this paper. All authors read and approved the final manuscript.

Conflicts of Interest: "The authors declare no conflict of interest."

\section{References}

[1] Wang, Z. G., Liu, Z. H., \& Xiang, R. G. (2011). Some criteria for meromorphic multivalent starlike functions. Applied Mathematics and Computation, 218(3), 1107-1111.

[2] Goswami, P., Bulboacă, T., \& Alqahtani, R. T. (2016). Simple sufficient conditions for starlikeness and convexity for meromorphic functions. Open Mathematics, 14(1), 557-566.

[3] Jack, I. S. (1971). Functions starlike and convex of order $\alpha$. Journal of the London Mathematical Society, 2(3), 469-474.

(C) 2020 by the authors; licensee PSRP, Lahore, Pakistan. This article is an open access article distributed under the terms and conditions of the Creative Commons Attribution (CC-BY) license (http://creativecommons.org/licenses/by/4.0/). 